\title{
Comparisons of judgments using rank ordering and regression models
}

\author{
J. MATTHEW MADDEN \\ Rensselaer Polytechnic Institute, Troy, New York 12181
}

\begin{abstract}
A comparison was made between judgments concerning values using rank ordering and a profile of value levels. Results suggest that the profile rating task and rank ordering measure different judgment processes. When a regression equation is computed for each subject, $\mathrm{R}^{2}$ ranges from very low to very high, indicating that some subjects do not make consistent value judgments. Further, values do not enter individual equations in a manner that would be predicted from ranked data. The value with the lowest average rank was second in terms of number of equations entered. Values with low rankings tend to enter equations with negative weights, indicating a strong influence of values with negative valences. Profile rating and regression analysis appear to provide more valid measures of value systems than rank ordering.
\end{abstract}

Rokeach (1971) reports research findings of considerable importance indicating highly significant and persistent changes in values obtained in $30-40 \mathrm{~min}$ in the classroom. As Rokeach points out (p. 458), these dramatic results have far-reaching implications for the fields of political science and propaganda, education, therapy, and ethics. The research, however, depends heavily on the ranking method. Aside from the limitations of the ordinal scale obtained when ranking is used, other aspects of ranking may merit reexamination in view of the potential impact of Rokeach's work.

One concern with the ranking method is that the subject focuses on one stimulus object at a time. He may make comparisons of that one stimulus with another stimulus or even more than one stimulus, but he is primarily judging single stimuli, one at a time. In more natural situations, judgments are based on a profile of a number of stimuli considered simultaneously. When making value judgments, a subject seems more likely to use a value system as a referent than individual values taken singly. There may be judgmental situations where only one value is relevant but these are probably rare. Individuals are more likely to base their judgments either on their entire value system, consisting of all the values they consider important, or on subsets of these values which may be considered as subsystems.

The multiple regression model may be used to study judgments of profiles. The general form is:

$$
\mathrm{Y}=\mathrm{W}_{0}+\mathrm{W}_{1} \mathrm{X}_{1}+\mathrm{W}_{2} \mathrm{x}_{2}+\cdots \mathrm{W}_{\mathrm{n}} \mathrm{X}_{\mathrm{n}}
$$

For our purposes, the variables $\mathrm{X}_{1}, \mathrm{X}_{2}, \cdots \mathrm{X}_{\mathrm{n}}$ are

Requests for reprints should be sent to the author, Department of Psychology, Rensselaer Polytechnic Institute, Troy, New York 12181. values. A matrix consisting of $\mathrm{N}$ values and $\mathrm{K}$ sets of levels on each of the values, or $K$ value profiles, can be generated. $\mathrm{Y}$ is a variable consisting of the judgments a subject makes for each profile, i.e., the similarity of each value profile or value system to his own. Solving the multiple regression equation, $\mathrm{R}^{2}$ indicates the consistency with which an individual makes his value judgments or the degree to which he possesses a value system. The variables (values) that enter the equation are those that constitute the individual's value system. The beta weights provide an indication of the relative importance of the values in any given value system, and a weight of zero associated with a value indicates that it is not a part of the individual's value system. Standard procedures can be used to detect nonlinear forms and interactions, if hypothesized. Thus, the multiple regression approach provides a rich array of data for comparison with findings obtained using the ranking method.

The multiple regression method has been utilized increasingly during the last decade. It has been applied to a wide range of subject matter ranging from selection of a harem (Christal, 1968), through job evaluation (Madden, 1964), to government policy on nuclear safeguards (Brady \& Rappoport, 1973). A comprehensive review is provided by Slovic and Lichtenstein (197i). Specifically, in the present context the multiple regression method will enable us to test a series of hypotheses: (a) If a subject rank orders a set of values, and if he then rates this set along with other sets, he should rate his own prior ranking or profile as high as or higher than any other and he should not rate any other profile higher than his own; (b) values ranked high (important) should enter the individual regression equations more often than those given the lower ranks; and (c) if a value enters a regression equation, it should have a positive weight. This follows from the basic nature of the ranking method where stimuli are ranked, 
Table 1

The Rank Ordering of the 18 Values Based on Mean Rank from the Second Experimental Session

\begin{aligned} & \hline Rank \multicolumn{1}{c}{ Value } \\ & \hline 1 Happiness \\ & 2 Self-respect \\ & 3 Freedom \\ & 4 Mature love \\ & 5 True friendship \\ & 6 Inner harmony \\ & 7 A sense of accomplishment \\ & 8 Wisdom \\ & 9 A world at peace \\ & 10 Family security \\ & 11 A comfortable life \\ & 12 Pleasure \\ & 13 An exciting life \\ & 14 Equality \\ & 15 A world of beauty \\ & 16 Salvation \\ & 17 Social recognition \\ & 18 National security \\ & \hline\end{aligned}

say, from most desirable to least desirable. The implication is that the subject is positively oriented toward the stimulus ranked least desirable, although less positively than toward the other stimuli.

\section{METHOD}

Subjects were students in an introductory psychology class consisting primarily of freshmen and sophomores. In the first experimental session, each subject provided demographic information and rank ordered 18 terminal values in terms of importance to the subject. A terminal value is defined as an end state of existence by Rokeach, and the set of 18 was taken from his 1971 paper. In the second experimental session, occurring 6 weeks after the. first, subjects again ranked the same set of 18 terminal values.

A set of 50 value profiles, each being a rank ordering of the 18 terminal values from the second session, was then randomly sampled from subjects participating in both experimental sessions. During the third experimental session, occurring 6 weeks after the second, subjects rated all 50 value profiles. The ratings were based on the degree to which each value profile matched the subject's own value system. A rating of 9 was given to a perfect match, 7 for an excellent match, 5 for a good match, 3 for a fair match, and 1 for a poor match. There were 146 subjects who participated in both the first and second session and 103 subjects participated in all three. Of the 146 subjects, 16 were females; of the 103 subjects, 13 were females.

\section{RESULTS}

The number of times each rank from 1 to 18 was assigned to each value was tabulated using the rank orderings from the second experimental session. Of the 324 cells in the resulting matrix, only 26 did not contain any entries. Three values, happiness, mature love, and national security, accounted for 13 of the empty cells. The mean rank for happiness was 1 , for mature love 4 , and for national security 18 . Thus, the distribution of rankings across values, with the exception of the three just mentioned, was rather flat and roughly rectangular, suggesting a high degree of variability.

A rank-order correlation between the rankings obtained in the first and second experimental sessions was computed for each subject. The range of rho was .18-.97, with a median of .78. When rho was computed between mean ranks, the result was .95 . Since the rho for mean ranks is often reported, it seems useful to note that in this case it would obscure the low individual rhos and the median rho appears to be a more conservative estimate of reliability. The rank ordering of the 18 values, based on mean rank from the second session, is given in Table 1 .

Table 2 gives the ratings assigned to their own profiles by the 34 subjects who participated in all three experimental sessions and whose profiles were sampled. Also in Table 2 are the total number of ratings given by subjects that were equal to or greater than the rating given their own profile and the average number of ratings equal to or greater than the subject's own profile rating, for each rating level. The grand mean is 7.21 , indicating that on the average these 34 subjects rated $14.5 \%$ of the 50 profiles equal to or greater than the rating they assigned to their own profile. Of the 245 "equal to" or "greater than" ratings, 119 , or $49 \%$, were "greater than."

As previously mentioned, a multiple regression equation was computed for each of 103 subjects. $R^{2}$ ranged from 10 (.01 stop criterion) to 86 and was distributed as given in Table 3 . Also in Table 3 is the mean number of predictors for each interval of $R^{2}$. Although the placement into categories of the values of $\mathbf{R}^{2}$ was arbitrary, a one-way analysis of variance was performed to test the significance of the differences among intervals. The resulting $\mathrm{F}=24.88, \mathrm{p}=.001$, with $\mathrm{df}=(3 / 99)$, at least suggests a significant difference in number of predictors as $R^{2}$ increases. Thus, as cognitive complexity increases, $R^{2}$ increases or, in other terms, as the complexity of the value system increases, so does $\mathrm{R}^{2}$.

In order to determine the relationship between consistency as measured by test-retest reliability and consistency as measured by $R^{2}$, the correlation

Table 2

Data for 34 Subjects on the Number of Times Ratings Equal to or Greater Than the Rating of the Subject's Own Profile Were Given

\begin{tabular}{cccc}
$\begin{array}{c}\text { Rating of } \\
\text { Own Profile }\end{array}$ & $\begin{array}{c}\text { Number of } \\
\text { Subjects }\end{array}$ & $\begin{array}{c}\text { Number of } \\
\text { Ratings } \geqslant \text { Own }\end{array}$ & Mean \\
\hline 9 & 2 & 0 & 0 \\
8 & 10 & 6 & .60 \\
7 & 8 & 44 & 5.50 \\
6 & 3 & 30 & 10.00 \\
5 & 6 & 61 & 10.17 \\
4 & 4 & 61 & 15.25 \\
3 & 1 & 43 & 43.00 \\
& 34 & 245 & 7.21 \\
\hline
\end{tabular}


Table 3

Distribution of $R^{2}$ and the Mean Number of Predictors for Each Interval

\begin{tabular}{ccc}
\hline $\mathrm{R}^{2}$ & $\begin{array}{c}\text { Number of } \\
\text { Subjects }\end{array}$ & $\begin{array}{c}\text { Mean Number } \\
\text { of Predictors }\end{array}$ \\
\hline $10-29$ & 19 & 1.58 \\
$30-49$ & 39 & 2.79 \\
$50-69$ & 38 & 3.53 \\
$70-89$ & 7 & 4.86 \\
\hline
\end{tabular}

Table 4

Number of Times Each Value Entered on Equation, Rank Ordering Based on that Number, and Mean Rank From the Second Session

\begin{tabular}{cccc}
\hline & Number of & \multicolumn{2}{c}{ Mean Rank } \\
Value & Entries & Entries & Ranking \\
\hline 1 & 15 & 10.5 & 11 \\
2 & 11 & 14 & 13 \\
3 & 15 & 10.5 & 6 \\
4 & 15 & 10.5 & 9 \\
5 & 8 & 16 & 15 \\
6 & 13 & 13 & 14 \\
7 & 15 & 10.5 & 10 \\
8 & 10 & 15 & 7 \\
9 & 21 & 4 & 1 \\
10 & 16 & 7 & 5 \\
11 & 33 & 3 & 2 \\
12 & 16 & 7 & 18 \\
13 & 5 & 18 & 12 \\
14 & 39 & 1 & 16 \\
15 & 7 & 17 & 17 \\
16 & 34 & 2 & 4 \\
17 & 16 & 7 & 3 \\
18 & 19 & 5 & 8 \\
\hline
\end{tabular}

between the rho for the first two experimental sessions and $\mathrm{R}^{2}$ was computed, resulting in $\mathrm{r}=.08$. For an $\mathrm{N}$ of 103 , this value is not significant, indicating that rho and $\mathbf{R}^{2}$ measure different kinds of consistency.

Table 4 gives, for each value, the number of times it entered an equation, the rank ordering in terms of the number of times entering an equation, and the mean rank obtained in the second experimental session. There are some rather striking differences, such as that in Value 14-salvation. The rank-ordering data indicate a rank of 16 , while the multiple regression data give a rank of 1. For Value 12, national security, the rank data result in a rank of 18 , the regression data 7 . Other interesting differences are found in Value 8-freedomranking $=7$ but regression $=15$, and in Value 13pleasure-ranking $=12$ but regression $=18$. Finally, for Value 3-a sense of accomplishment-ranking $=6$, regression $=10.5$. For these values, subjects evidently engaged in a different sort of cognitive process in the more natural situation than when ranking. On the other hand, for the remaining values, the outcomes are quite similar. Although the rho of .72 for these data is statistically significant, it obscures these differences.

Table 5 gives three pieces of information associated with those values assigned a rank order of $1,2, \ldots 18$.
The first, Column 2, is the number of times values assigned each rank entered regression equations with a positive weight; Column 3 gives the corresponding numbers for negative weights. Column 4 gives the rank ordering in terms of the number of times each value entered an equation. In other words, going across the first line, of the 103 values ranked first by the 103 subjects, 66 of them entered an equation with a positive weight, 1 entered with a negative weight, and the rank in terms of number of equations entered is Number 1 . The most striking thing about Table 5 is the bottom line. The variable ranked last, 18 th, ranked Number 2 in terms of the number of equations entered, and all weights were negative. Of the 308 equation entries, 187 of the weights were positive and 121 were negative. Although there is no method for estimating the expected number of negative coefficients, $39 \%$ seems an unusually large proportion.

\section{DISCUSSION}

If it can be assumed that $5 \%$ of the profile ratings would be equal to or greater than the rating subject's gave to their own profile on a chance basis, then the finding of $14.5 \%$ may be viewed as a significant one. Further, $7 \%$ of the ratings given by the 34 subjects whose data are in Table 2 were higher than the rating given to their own profiles. The assumption of $5 \%$ seems a conservative one, especially when the rather high testretest reliabilities are considered. If a subject ranks the 18 values reliably on two occasions, it seems reasonable to expect that he would recognize his ranking when viewed as a profile, even when other profiles are present. Having recognized his own profile, he should, by definition, give it a rating of 9 . Only 2 of the 34 subjects did this. It appears, therefore, that the profile rating task is measuring a different entity than the ranking task.

Table 5

For Each Rank Assigned a Value by Each Subject, the Number of Times an Equation Was Entered With a Positive Weight, With a Negative Weight, and the Rank Order in Terms of the Total Number of Equations Entered

\begin{tabular}{cccc}
\hline $\begin{array}{c}\text { Rank by } \\
\text { Subject }\end{array}$ & $\begin{array}{c}\text { Number of } \\
\text { Positive } \\
\text { Entries }\end{array}$ & $\begin{array}{c}\text { Negative } \\
\text { Entries }\end{array}$ & $\begin{array}{c}\text { Rank Number } \\
\text { of Entries }\end{array}$ \\
\hline 1 & 66 & 1 & 1 \\
2 & 26 & 1 & 3 \\
3 & 15 & 0 & 6 \\
4 & 15 & 1 & 5 \\
5 & 15 & 6 & 4 \\
6 & 10 & 3 & 8 \\
7 & 9 & 3 & 11 \\
8 & 10 & 3 & 8 \\
9 & 6 & 6 & 11 \\
10 & 2 & 4 & 18 \\
11 & 4 & 5 & 17 \\
12 & 2 & 9 & 13.5 \\
13 & 4 & 9 & 8 \\
14 & 1 & 9 & 15.5 \\
15 & 1 & 11 & 11 \\
16 & 0 & 10 & 15.5 \\
17 & 1 & 10 & 13.5 \\
18 & 0 & 30 & 2 \\
& 187 & 121 & \\
\hline
\end{tabular}


Coupled with the lack of correlation between test-retest reliability and $\mathrm{R}^{2}$, we have evidence that either the ranking or the profile rating task is measuring subject's value systems, but not both.

The data in Table 3 might be expected on an intuitive basis. These data suggest that, for some subjects, judgments are very inconsistent and that their value systems are not yet well formed. At the other end of the continuum, some subjects are very consistent and have well-formed value systems. The majority of the subjects appear to be in a developmental stage between the two extremes. The ranking method, of course, is not sensitive to this kind of phenomenon. After the ranking task is accomplished, results are treated as if all subjects possessed a value system that includes all the values on the list and that constitutes the basis for consistent judgment.

The rank ordering of the values can be viewed as a form of subjective weighting. The data in Table 4 , from this point of view, indicate that for some values, subjects' subjective weights differ widely from the weights they actually use, considering the simple case of weights of zero or not zero. This finding is similar to that of Valenzi and Andrews (1973) using data from an employment interviewing situation. Thus, when ranking subjects select national security on the average as the least important, but it is 7 th in terms of its actual use. Applying this interpretation to the other differences reported above, we have a strong indication that subjects are unable to identify those values they actually use in making value judgments to a highly significant degree. In other words, they are unable to solve a multiple regression equation mentally. If this interpretation is correct, inferences from ranked data must be made with extreme care.

Further evidence of the weakness of the ranking method is found in Table 3. Of 103 subjects who ranked some value as 1 , in only 66 cases did the value ranked 1 come into an equation. For the value ranked 2 , an entry into an equation occurred only 26 times, and so on. What is even more striking is that the value ranked last (18) entered equations 30 times, the second most frequent, as indicated in the last column. If a subject assigns a value the highest relative weight when he ranks it 1 , and so on down to the lowest relative weight when he ranks it 18, what he does when he makes a value judgment is considerably different from what he thinks he does. The implication here is that a subject cannot depict his value system by ranking. He is influenced, perhaps by such factors as social acceptability, conformity pressures, and others not now known. Another possibility is that values have more complex attributes than assumed in the past. That is, for instance, an individual may have formed a value over a long period of time during which it acquired a very strong positive valence. During his college experience, he may be in the process of questioning both the strength and the sign of that valence. When making a judgment concerning only that single value (as in ranking), the current, more transitory questioning process may result in a low ranking. However, in the more natural profile judgment situation, the deeper or older attributes of the value dominate the judgment even though the individual is not aware of what is happening.

Another interesting aspect of value judgments, considering the data in Table 5, is that values ranked low not only come into equations frequently, but the weights associated with them are negative. Thus, subjects seem to make their judgments on the basis of anti-values, or values which possess a negative valence, more than half as often as their judgments are based on values with a positive valence. The presence of a negatively regarded value, then, appears to be an important influence in the making of value judgments. For the measurement of value systems it would seem to be essential that this effect be taken into account and the measurement procedure be sensitive to both value domains.

\section{REFERENCES}

Brady, D., \& Rappoport, E. Policy-capturing in the field: The nuclear safeguards problem. Organizational Behavior and Human Performance, 1973, 9, 253-266.

Christal, R. E. Selecting a harm-and other applications of the policy-capturing model. Journal of Experimental Education, 1968, 36, 35-41.

MADDEN, J. M. A policy-capturing model for analyzing individual and group judgment in job evaluation. Journal of Ind ustrial Psychology, 1964, 2, 36-42.

ROKEACH, M. Long-range experimental modification of values, attitudes and behavior. American Psychologist, 1971, 26, 453-459.

Slovic, P., \& Lichtenstein, S. C. Comparison of Bayesian and regression approaches to the study of information processing in judgment. Organizational Behavior and Human Performance, $1971,6,649-744$

VAlEnZI, E., \& ANDREwS, I. R. Individual differences in the decision process of employment interviewers. Journal of Applied psychology, 1973, 58, 49-53.

(Received for publication November 14, 1975.) 